\title{
Caracterização da Consulta de Enfermagem na Atenção à Pessoa com Hipertensão e Diabetes
}

Characterization of the Nursing Consultation in the Care of the Person With Hypertension and Diabetes

Caracterización de la Enfermería en la Consulta que Atienden a Personas con Hipertensión y Diabetes

Clemilson Sousa Silva ${ }^{1}$

Alecsandra Ferreira Tomaz ${ }^{2}$

Wezila Gonçalves do Nascimento ${ }^{3}$

Ana Patrícia Taveira Silva ${ }^{4}$

Jairo Porto Alves 5

\section{Resumo}

Objetivo: Descrever as ações dos enfermeiros através da consulta de enfermagem instrumentalizada pela SAE à pacientes hipertensos e diabéticos nos Centros de Saúde. Método: estudo de caráter descritivo, transversal, de abordagem quantitativa, com amostra de 10 enfermeiros. Utilizou-se um questionário contemplando as etapas da Sistematização da Assistência de Enfermagem. Resultados: todos os enfermeiros realizam exame físico, $70 \%$ utilizam protocolos e $90 \%$ registram as consultas. Do total, $60 \%$ não conseguem classificar os diagnósticos de acordo com as prioridades; $70 \%$ não desenvolvem grupos educativos, $100 \%$ prescrevem exames e avaliam o pé diabético; 70\% mensuramos resultados

${ }^{1}$ Graduado em Enfermagem pela Universidade Estadual da Paraíba. Pós-graduado em Enfermagem do Trabalho pela Universidade Norte do Paraná. Autor correspondente: R. Baraúnas, 351 - Universitário. CEP 58429-500. Campina Grande - PB. E-mail: clemilsonpb15@gmail.com

${ }^{2}$ Graduada em Fisioterapia pela Universidade Federal da Paraíba. Mestrado em Engenharia de Produção pela Universidade Federal da Paraíba.

${ }^{3}$ Graduada em Enfermeira pela Universidade Federal da Paraíba e Especialista em Saúde Coletiva.

${ }^{4}$ Graduada em Enfermagem pela Universidade Estadual da Paraíba.

${ }^{5}$ Graduando em Enfermagem pela Universidade Estadual da Paraíba.

Recebido: Nov/2016 - Aceito: Fev/2017. 
e $80 \%$ percebem a sua eficácia.

Conclusão: Os enfermeiros abordados no estudo têm um bom ponto de partida com a realização do exame físico, porém, mais da metade dos enfermeiros investigados não ultrapassa a etapa do diagnóstico, comprometendo as demais etapas da SAE.

Descritores: Enfermagem no Consultório; Cuidados de Enfermagem; Hipertensão; Diabetes Mellitus.

\section{Abstract}

Objective: To describe the actions of nurses through the nursing consultation instrumentalized by $S A E$ to hypertensive and diabetic patients in the Health Centers. Methods: descriptive study, cross-sectional, quantitative approach, with sample of 10 nurses. We used a questionnaire addressing the stages of systematization of nursing care. Results: all nurses perform physical examination, $70 \%$ use protocols and $90 \%$ record queries. Of the total, $60 \%$ fail to classify diagnoses according to the priorities; $70 \%$ do not develop educational groups, $100 \%$ prescribe tests and evaluate the diabetic foot; $70 \%$ measure the results and $80 \%$ realize their effectiveness. Conclusion: The nurses covered in the study have a good starting point with the physical examination, however, more than half of the nurses investigated do not go beyond the diagnostic stage, compromising the other stages of the $S A E$.

Descriptors: Office Nursing; Nursing Care; Hypertension; Diabetes Mellitus.

\section{Resumen}

Objetivo: Describir las acciones de las enfermeras mediante la consulta de enfermería manipulado por el SAE hipertensos y pacientes diabéticos en los centros de salud.. Métodos: Estudio descriptivo, transversal, enfoque cuantitativo, con la muestra de 10 enfermeras. Se utilizó un cuestionario de abordar las etapas de la sistematización de la asistencia de enfermería. Resultados: todas las enfermeras realizan el examen físico, los protocolos de uso de $70 \%$ y 90 consultas de registro\%. Del total, 60\% de fracaso para clasificar los diagnósticos de acuerdo com las prioridades; $70 \%$ no desarrollan grupos educativos, $\quad 100 \%$ prescribirpruebas y evaluarel pie diabético; $70 \%$ medir los resultados y el 
$80 \%$ da cuenta de sueficacia.

Conclusión: El estúdio de las enfermeras es un buenpunto de partida con el examen físico, sin embargo, más de la mitad de las enfermeras investigadas no va más allá de la etapa de diagnóstico, comprometer los pasos restantes de la SAE.

Descriptores: Enfermería de Consulta; Atención de Enfermería; Hipertensión; Diabetes Mellitus.

\section{Introdução}

O Diabetes Mellitos (DM) e a Hipertensão Arterial Sistêmica (HAS) constituem os principais fatores de risco populacional para as doenças cardiovasculares, motivo pelo qual constituem agravos de saúde pública onde cerca de 60 a $80 \%$ dos casos podem ser tratados na rede básica ${ }^{(1)}$.

$$
\text { Estudos epidemiológicos }
$$
realizados pelo Ministério da Saúde (MS) desde 2003 indicam que o DM e HAS são condições que geralmente estão associadas. A prevalência de hipertensão é de aproximadamente o dobro entre os diabéticos em comparação com os não diabéticos. Porém, a hipertensão afeta $40 \%$ ou mais dos indivíduos diabéticos ${ }^{(2)}$.
Compreende-se o Diabetes Mellitos como uma síndrome de causas múltiplas, oriunda da falta de insulina ou da incapacidade desta de exercer suas funções e efeitos adequados dentro do organismo, relacionando-se a distúrbios no metabolismo dos carboidratos, proteínas e lipídios. A Hipertensão Arterial Sistêmica é definida como uma pressão arterial sistólica maior ou igual a $140 \mathrm{mmHg}$ e uma pressão arterial diastólica maior ou igual a $90 \mathrm{mmHg}$, em indivíduos que não estão fazendo uso de medicação anti-hipertensiva ${ }^{(3)}$.

Uma recomendação do Ministério da Saúde chama a atenção para o fato de que não se deve apenas valorizar os níveis de pressão arterial, mas é necessária uma avaliação do risco cardiovascular global $^{(3)}$.

Nesse contexto, o enfermeiro, por meio de sua formação e qualificação profissional, contribui para a melhoria do atendimento aos usuários hipertensos e diabéticos, viabilizando a assistência prestada e diminuindo as causas que comprometem a qualidade e a agilidade dos serviços oferecidos ${ }^{(4)}$.

A consulta de enfermagem (CE), utilizada pelo profissional enfermeiro na Atenção Primária à Saúde (APS) promove o vínculo entre enfermeiro e usuário para identificar os problemas, envolvendo o processo saúde-doença, 
através das prescrições e implementações de enfermagem que colaboram para promoção, proteção, recuperação ou reabilitação do usuário com amparo legal, mediante a Lei do Exercício Profissional da Enfermagem de $n^{\mathrm{o}} 7.498 / 86^{(5)}$.

Ainda nesse sentido, em termos legais, a Resolução 159/1993 do Conselho Federal de Enfermagem $(\mathrm{COFEN})^{(6)}$ considera que a consulta de enfermagem tem como fundamento os princípios de universalidade, equidade, resolutividade e integralidade das ações de saúde. Essa mesma Resolução no seu artigo $1^{\circ}$ afirma que a consulta de enfermagem deve ser obrigatoriamente desenvolvida na assistência de enfermagem em todos os níveis de assistência à saúde, seja em instituição pública ou privada.

A CE objetiva desenvolver uma visão holística, captando as informações necessárias advindas do usuário, levando ao diagnóstico preciso e possibilitando elaborar um plano de assistência que seja condizente com a necessidade identificada. Através desse instrumento, o profissional tem a oportunidade de prestar um atendimento individual dando ênfase a educação dos pacientes quanto à condição clínica em que encontram ${ }^{(7)}$.
$\mathrm{Na}$ prática, a $\mathrm{CE}$ viabiliza o trabalho do enfermeiro no atendimento ao usuário e permite a identificação de problemas e as decisões a serem tomadas a partir dela. Portanto, ela deve ser norteada através da Sistematização da Assistência de Enfermagem (SAE), método científico com aplicação específica, de modo que o cuidado seja direcionado de forma adequada e efetiva $^{(8)}$.

$\mathrm{O}$ processo de enfermagem ou a sistematização da assistência de enfermagem é composto por um modelo sistemático, em cinco etapas para tomada de decisões clínicas que incluem: coleta de dados, diagnóstico, planejamento, implementação e avaliação $^{(9)}$.

A investigação/histórico é o primeiro passo do processo que consiste na coleta de dados, e tem como objetivo de descrever o perfil do estado de saúde, das funções e dos componentes relacionados à saúde do usuário, da família ou de uma comunidade. No diagnóstico a prioridade desta é um tipo de raciocínio crítico que os profissionais enfermeiros devem desenvolver durante a consulta. A prioridade deve ser estabelecida com base nas necessidades dos indivíduos, família e comunidade $^{(10)}$. 
O planejamento implica no estabelecimento dos objetivos da assistência, analisar as consequências que poderiam advir de diferentes atuações, optar entre alternativas, determinar metas específicas a serem atingidas. A implementação, coloca-se em prática o plano de ação, investigando a situação atual e, se necessário, alterar o planejamento. A etapa final do processo é a avaliação, crucial para determinar se a condição ou o bem- estar do usuário melhorou após a aplicação do processo de enfermagem. O enfermeiro desenvolve e acompanha medidas avaliativas para determinar se atingiu os resultados esperados ${ }^{(9)}$.

No Brasil, o modelo mais conhecido e utilizado para a implantação da SAE é o proposto e desenvolvido por Horta, de forma que esse modelo se baseia no método de tomada de decisões que se sustenta nos métodos científicos ${ }^{(11)}$.

A aplicação da SAE nas instituições de saúde apresenta os seguintes aspectos positivos: segurança no planejamento, execução e avaliação das condutas de enfermagem, a individualização da assistência, visibilidade e autonomia para o enfermeiro $^{(12)}$.

Diante desse contexto, o presente estudo foi desenvolvido com o objetivo de descrever as ações dos enfermeiros através da consulta de enfermagem instrumentalizada pela SAE à pacientes hipertensos e diabéticos nos Centros de Saúde.

\section{Método}

Trata-se de uma pesquisa de caráter descritivo, de abordagem quantitativa e exploratória. $\mathrm{O}$ estudo foi realizado em todos os Centros de Saúde na cidade de Campina Grande/PB, exceto um que não foi incluído na pesquisa por não desenvolver ações do Programa Hiperdia. A coleta de dados deu-se entre os meses de novembro de 2014 a Janeiro de 2015.

A amostra da pesquisa foi intencional, sendo selecionados os enfermeiros que realizam acompanhamento dos usuários hipertensos e diabéticos nos Centros de Saúde deste município, no total de 10 sujeitos. O critério de inclusão é que o profissional deveria ser enfermeiro e que desenvolvesse ações referentes ao Programa Hiperdia nos Centro de Saúde. O critério de exclusão abrangeu apenas aqueles enfermeiros afastados/de licença.

Para a coleta de dados utilizou-se um questionário composto de vinte e cinco questões objetivas, de múltipla 
escolha, direcionado, numa abordagem que contemplasse as cinco etapas da SAE, com ênfase nas seguintes áreas: acolhimento, aspectos clínicos das doenças, cuidados gerais ao paciente, promoção e prevenção da saúde.

Para análise dos dados foi utilizada estatística descritiva. O banco de dados foi elaborado através do programa Excel (Microsoft 2010). Os dados são apresentados sob a forma de frequências absoluta e relativa.

Neste estudo, foram levadas em consideração as recomendações preconizadas na Resolução n466 de 12 de dezembro de 2012 do Conselho Nacional de Saúde/MS. A pesquisa foi aprovada pelo Comitê de Ética em Pesquisa da Universidade Estadual da Paraíba, sob protocolo $\mathrm{n}^{\circ}$ 38501514.0 .0000 .5187$.

\section{Resultados}

Ao caracterizar o perfil dos enfermeiros que trabalham com o Hiperdia nos Centros de Saúde de Campina Grande/PB, percebe-se que $90 \%$ são do sexo feminino; $50 \%$ do total com mais de 10 anos de profissão e $80 \%$ deles informaram que possuem pós-graduação em Saúde Pública.

$\mathrm{Na}$ investigação, primeira etapa da SAE, constatou-se que $100 \%$ fazem uso do exame físico para a busca de informações e estabelecimento da condição clínica dos usuários. Os principais fatores de riscos encontrados foram: o sobrepeso com $60 \%$ seguido de lesão de Membros inferiores (MMII) ou ocular com $30 \%$.

Os protocolos são também utilizados durante a $\mathrm{CE}$, sendo que mais de $50 \%$ dos enfermeiros investigados utilizam os Manuais do Ministério da Saúde (MS), seguido da utilização do Índice de Massa corpórea (IMC), com $42,9 \%$, como instrumento para avaliar os fatores de riscos que levam a complicações da Hipertensão Arterial Sistêmica e o Diabetes Mellitus, segundo a tabela 1 .

Durante o atendimento, o enfermeiro deve registrar o máximo de informações possíveis. Nota-se que $90 \%$ da amostra consegue registrar as informações, sendo estas anotadas no prontuário e no cartão do usuário, com $44,4 \%$ cada. Entre os enfermeiros estudados, $\quad 60 \%$ informaram dificuldades para realizarem a CE. As principais dificuldades relatadas foram: ausência de recursos; falta de tempo e ausência de treinamento com 83,3\%, $66,7 \%$ e $50 \%$ respectivamente, conforme a tabela 1 . 
Tabela 1: Distribuição das Ações que Caracterizam a Etapa da Investigação da SAE por Enfermeiros dos Centros de Saúde.

\begin{tabular}{|c|c|c|c|c|c|c|c|}
\hline \multirow{3}{*}{ Variáveis da Investigação } & \multirow{2}{*}{\multicolumn{2}{|c|}{ SIM }} & \multirow{2}{*}{\multicolumn{2}{|c|}{ NÃO }} & \multirow{3}{*}{ Justificativa } & \multirow[b]{3}{*}{$\mathrm{N}$} & \multirow[b]{3}{*}{$\%$} \\
\hline & & & & & & & \\
\hline & $\mathrm{N}$ & $\%$ & $\mathrm{~N}$ & $\%$ & & & \\
\hline \multirow{4}{*}{$\begin{array}{l}\text { Durante a consulta você } \\
\text { utiliza o Exame Físico para } \\
\text { detectar Fatores de Riscos } \\
\text { no usuário Hipertensos e/ou } \\
\text { Diabéticos? }\end{array}$} & 10 & 100 & 0 & 0 & \multicolumn{2}{|l|}{ Fatores de riscos encontrados? } & \\
\hline & & & & & Sinais vitais alterados & 2 & 20 \\
\hline & & & & & Sobrepeso & 6 & 60 \\
\hline & & & & & $\begin{array}{l}\text { Lesão de Membros inferiores ou } \\
\text { ocular }\end{array}$ & 3 & 30 \\
\hline $\begin{array}{l}\text { Você utiliza alguma } \\
\text { escala/protocolo para avaliar } \\
\text { o risco de complicações dos }\end{array}$ & 7 & 70 & 3 & 30 & Quais protocolos utilizados? & & \\
\hline \multirow[t]{3}{*}{ Hipertenso/Diabético? } & & & & & Manual do Ministério da Saúde & 4 & 57,1 \\
\hline & & & & & $\mathrm{IMC}$ & 3 & 42,9 \\
\hline & & & & & Roteiro próprio & 1 & 14,3 \\
\hline $\begin{array}{l}\text { Durante a consulta você } \\
\text { registra os achados e } \\
\text { cuidados realizados aos }\end{array}$ & 9 & 90 & 1 & 10 & $\begin{array}{l}\text { Onde são realizados os } \\
\text { registros? }\end{array}$ & & \\
\hline \multirow[t]{3}{*}{ Hipertensos e Diabéticos? } & & & & & Prontuário & 4 & 44,4 \\
\hline & & & & & Cartão do Usuário & 1 & 11,1 \\
\hline & & & & & Ambos & 4 & 44,4 \\
\hline \multirow{6}{*}{$\begin{array}{l}\text { Existe alguma dificuldade } \\
\text { para a realização da consulta } \\
\text { de enfermagem? }\end{array}$} & 6 & 60 & 4 & 40 & Quais dificuldades? & & \\
\hline & & & & & Falta de tempo & 4 & 66.7 \\
\hline & & & & & Ausência de recursos & 5 & 83.3 \\
\hline & & & & & $\begin{array}{l}\text { Falta de interesse do usuário } \\
\text { pela unidade }\end{array}$ & 2 & 33.3 \\
\hline & & & & & $\begin{array}{l}\text { Ausência de treinamento e } \\
\text { conhecimento dos protocolos } \\
\text { Dificuldade de acesso aos } \\
\text { protocolos/manuais }\end{array}$ & 3 & 50 \\
\hline & & & & & & 1 & 16,7 \\
\hline
\end{tabular}

Fonte: Dados da pesquisa, 2015.

$\mathrm{Na}$ segunda etapa da SAE, o enfermeiro, de posse das informações do usuário, identifica e estabelece os diagnósticos de enfermagem de acordo com a condição clínica investigada. No presente estudo, apenas $40 \%$ consegue desenvolver os diagnósticos; e a principal dificuldade relatada foi a não existência da North American Nursing Diagnosis Association/Classificação Internacional para a Prática de Enfermagem (NANDA/CIPE), com $80 \%$, conforme a tabela 2 . 
Tabela 2 - Distribuição das Ações que Caracterizam a Etapa Do Diagnóstico da SAE por Enfermeiros dos Centros de Saúde.

\begin{tabular}{|c|c|c|c|c|c|c|c|}
\hline \multirow[t]{2}{*}{ Variáveis do Diagnóstico } & \multicolumn{2}{|c|}{ SIM } & \multicolumn{2}{|c|}{$\mathrm{NÃO}$} & \multirow[t]{2}{*}{ Justificativa } & \multirow[b]{2}{*}{$\mathrm{N}$} & \multirow[b]{2}{*}{$\%$} \\
\hline & $\mathrm{N}$ & $\%$ & $\mathrm{~N}$ & $\%$ & & & \\
\hline \multirow[t]{4}{*}{$\begin{array}{l}\text { Você consegue traçar com } \\
\text { frequência os diagnósticos } \\
\text { de enfermagem? }\end{array}$} & 4 & 40 & 6 & 60 & $\begin{array}{l}\text { Principais dificuldades } \\
\text { Não existe NANDA/CIPE* }\end{array}$ & 8 & 80 \\
\hline & & & & & $\begin{array}{l}\text { Poucas informações passadas } \\
\text { pelo usuário }\end{array}$ & 4 & 40 \\
\hline & & & & & Falta de tempo & 5 & 50 \\
\hline & & & & & Falta de capacitação & 4 & 40 \\
\hline $\begin{array}{l}\text { Você consegue classificar os } \\
\text { Diagnósticos de acordo com } \\
\text { suas prioridades? }\end{array}$ & 2 & 20 & 6 & 60 & & & \\
\hline
\end{tabular}

$\mathrm{Na}$ fase de planejamento buscouse investigar se o enfermeiro consegue desenvolver estratégias para que $\mathrm{o}$ usuário tenha a adesão medicamentosa satisfatória e consciência da importância do autocuidado na busca de mudança de estilo de vida. Verificou-se que $100 \%$ deles relataram que utilizam estratégias para mudar estilo de vida através da dieta saudável e monitoramento de peso, segundo a tabela 3 .

Na mesma tabela observa-se que o autocuidado é estimulado por todos da amostra, e dentre as estratégias mais utilizadas encontra-se a orientação da alimentação saudável, mencionada por $100 \%$ da amostra. Cuidados com o pé diabético e uso do calçado adequado também foi estimulado por $90 \%$ dos enfermeiros.

$\mathrm{Na}$ implementação, quarta etapa da SAE, observa-se na tabela 4 que apenas $\quad 30 \%$ dos enfermeiros informaram sobre a existência de grupos de educação para a saúde no Centro de Saúde onde trabalham; porém $100 \%$ deles solicitam exames para os hipertensos e diabéticos durante a consulta.

No decorrer da consulta, especificamente na realização do exame físico todos avaliam a condição do pé diabético, quanto às lesões, estado das unhas, calos, deformidades e tipo de calçado utilizado. 
Tabela 3 - Distribuição das Ações que Caracterizam a Etapa do Planejamento da SAE por Enfermeiros dos Centros de Saúde.

\begin{tabular}{|c|c|c|c|c|c|c|c|}
\hline \multirow[t]{2}{*}{ Variáveis do Planejamento } & \multicolumn{2}{|c|}{ SIM } & \multicolumn{2}{|c|}{ NÃO } & \multirow[t]{2}{*}{ Justificativa } & \multirow[b]{2}{*}{$\mathbf{N}$} & \multirow[b]{2}{*}{$\%$} \\
\hline & $\mathrm{N}$ & $\%$ & $\mathrm{~N}$ & $\%$ & & & \\
\hline $\begin{array}{l}\text { Você utiliza } \text { estratégias } \\
\text { para estimular mudanças } \\
\text { no estilo de vida dos } \\
\text { pacientes com Hipertenso } \\
\text { /Diabético? }\end{array}$ & 10 & 100 & 0 & 0 & $\begin{array}{l}\text { Quais estratégias? } \\
\text { Adesão medicamentosa } \\
\text { Dieta saudável } \\
\text { Atividade física } \\
\text { Monitorar peso } \\
\text { Diminuir consumo de álcool } \\
\text { e cigarro }\end{array}$ & $\begin{array}{l}9 \\
10 \\
8 \\
10 \\
9\end{array}$ & $\begin{array}{l}90 \\
100 \\
80 \\
100 \\
90\end{array}$ \\
\hline $\begin{array}{l}\text { Em relação a adesão } \\
\text { medicamentosa, é } \\
\text { realizado algum tipo de } \\
\text { intervenção? }\end{array}$ & 9 & 90 & 1 & 10 & $\begin{array}{l}\text { Quais intervenções? } \\
\text { Atividade de educ. e saúde } \\
\text { Incentiva e orienta o uso } \\
\text { Conversa sobre os efeitos } \\
\text { Visita domiciliar } \\
\text { Estimula o ACS para } \\
\text { abordar na visita } \\
\text { Orienta melhor horário para }\end{array}$ & $\begin{array}{l}5 \\
9 \\
8 \\
4 \\
4\end{array}$ & $\begin{array}{l}55,6 \\
100 \\
88,9 \\
44,4 \\
44,4\end{array}$ \\
\hline $\begin{array}{l}\text { Você estimula o } \\
\text { autocuidado durante a }\end{array}$ & 10 & 100 & 0 & 0 & $\begin{array}{l}\text { uso } \\
\text { Quais estímulos ao } \\
\text { autocuidado? }\end{array}$ & & \\
\hline Diabéticos? & & & & & $\begin{array}{l}\text { Adesão medicamentosa } \\
\text { Alimentação saudável } \\
\text { Monitorar PA e glicemia } \\
\text { Cuidado com o pé diabético } \\
\text { Manter peso ideal } \\
\text { Abandono do álcool e tabaco } \\
\text { Uso de calçado adequado }\end{array}$ & $\begin{array}{l}9 \\
10 \\
10 \\
9 \\
10 \\
10 \\
9\end{array}$ & $\begin{array}{l}90 \\
100 \\
100 \\
90 \\
100 \\
100 \\
90\end{array}$ \\
\hline $\begin{array}{l}\text { Vocêutiliza algum critério } \\
\text { para o reagendamento } \\
\text { desses usuários? }\end{array}$ & 8 & 80 & 2 & 20 & & & \\
\hline
\end{tabular}

Fonte: Dados da pesquisa, 2015.

Orientam também os usuários que fazem uso de insulina, sobre o rodízio do local de aplicação, seu armazenamento, o consumo de carboidratos e açúcares, realização de exames de sangue e urina e teste de tolerância à glicose, quando necessário.

$\mathrm{Na}$ quinta e última etapa da SAE pode-se observar que $70 \%$ dos enfermeiros conseguem avaliar os resultados alcançados mediante as intervenções estabelecidas junto ao usuário hipertenso e/ou diabético; para tanto o enfermeiro utiliza como instrumento a consulta, os exames e os registros. Um total de $80 \%$ percebe eficácia nos resultados através da implementação dos cuidados, conforme tabela 5 a seguir. 
Tabela 4 - Distribuição das Ações que Caracterizam a Etapa da Implementação da SAE por Enfermeiros dos Centros de Saúde.

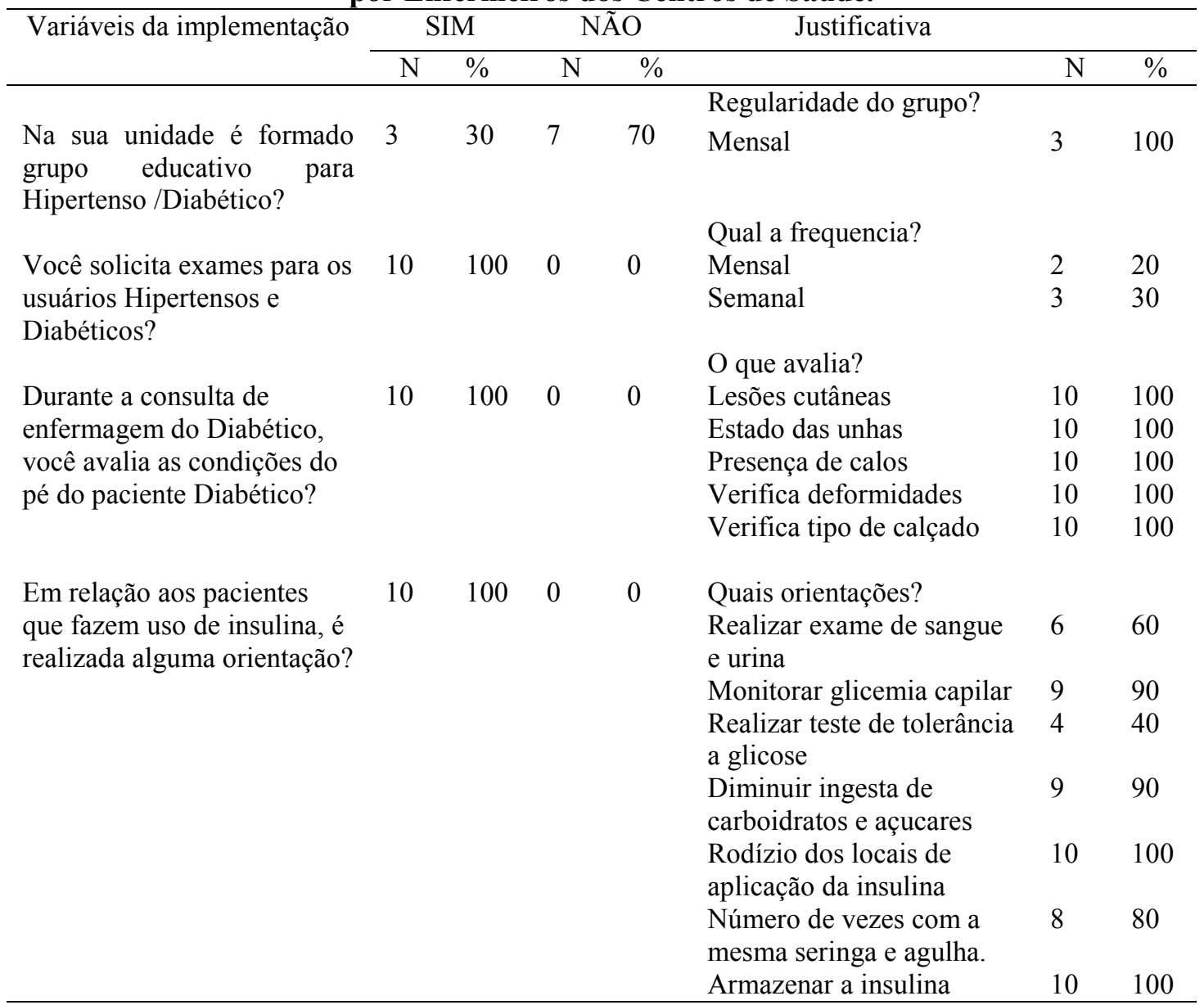

Fonte: Dados da pesquisa, 2015.

Nem sempre os resultados

esperados diante das implementações

dos cuidados resultam positivamente, e isso faz com que o enfermeiro busque outras condutas para serem direcionadas junto ao usuário.

Tabela 5 - Distribuição das Ações que Caracterizam a Etapa da Avaliação Da SAE Por Enfermeiros Dos Centros De Saúde.

\begin{tabular}{|c|c|c|c|c|c|c|c|}
\hline \multirow[t]{2}{*}{ Variáveis da Avaliação } & \multicolumn{2}{|c|}{ SIM } & $\mathrm{NA \tilde {A }}$ & & Justificativa & \multirow[b]{2}{*}{$\mathrm{N}$} & \multirow[b]{2}{*}{$\%$} \\
\hline & $\mathrm{N}$ & $\%$ & $\mathrm{~N}$ & $\%$ & & & \\
\hline $\begin{array}{l}\text { Você consegue mensurar ou avaliar } \\
\text { os resultados alcançados mediante }\end{array}$ & 7 & 70 & 1 & 10 & $\begin{array}{l}\text { Através de que instrumento } \\
\text { é realizada a mensuração? }\end{array}$ & & \\
\hline as intervenções? & & & & & Consulta & 7 & 100 \\
\hline & & & & & Exames & 7 & 100 \\
\hline & & & & & Registros & 7 & 100 \\
\hline
\end{tabular}

Você percebe eficácia nos $\begin{array}{llll}8 & 80 & 1 & 10\end{array}$

resultados através da

implementação dos cuidados, junto

aos usuários Hipertensos

/Diabéticos da unidade a partir da

avaliação?

Fonte: Dados da pesquisa, 2015. 
$\mathrm{Na}$ pesquisa percebe-se que $40 \%$ dos enfermeiros reavaliam o usuário, $30 \%$ encaminham ao especialista, $10 \%$ fazem busca ativa para visualizar o que está ocorrendo para o resultado negativo e $20 \%$ não responderam a alternativa.

\section{Discussão}

Em relação ao perfil dos enfermeiros do estudo observa-se predominância do sexo feminino. Estudo sobre a atuação do enfermeiro no controle a hipertensão nas Unidades de Saúde da Família na cidade de Patos/PB, ${ }^{(13)}$ evidenciou uma característica já conhecida da enfermagem, que é a predominância das mulheres. Ressalta-se que os homens vêm adquirindo um espaço significante nessa profissão, com tendência crescente $^{(14)}$.

Percebe-se nessa pesquisa um percentual significativo de enfermeiros qualificados, corroborando com outros estudos em que se observou a crescente busca por parte dos enfermeiros por qualificação profissional, visando atender as exigências do mercado de trabalho ${ }^{(13)}$.

Todos os enfermeiros desse estudo afirmaram realizar o exame físico e a maioria utiliza os protocolos do MS para avaliar o risco de complicações. Resultados semelhantes foram encontrados em outro estudo em que a experiência na utilização dos instrumentos/protocolos na Consulta de Enfermagem (CE) foi eficiente quanto à forma e conteúdo, uma vez que possibilitou um olhar ampliado do processo saúde-doença, facilitando a atuação do enfermeiro na abordagem integral do paciente ${ }^{(8)}$.

Ainda sobre os fatores de risco, a pesquisa sobre a SAE fundamentada na CIPE e na teoria da adaptação em hipertensos, identificaram alguns dos fatores de risco para os pacientes cadastrados no HIPERDIA. Os autores ressaltam que os usuários sedentários eram hipertensos com maior frequência. Com isso, o entrecruzamento entre sedentarismo e condições clínicas indicou associação estatística apenas para Diabetes Mellitus e Diabetes Mellitus concomitante com Hipertensão Arterial $^{(15)}$.

No atual estudo, os enfermeiros utilizam alguma forma de registro das consultas, variando entre prontuário, cartão do usuário ou ambos. $\mathrm{O}$ registro no prontuário também foi verificado em outra pesquisa, com o intuito de fornecer a equipe multiprofissional, dados sobre a evolução do paciente; 
facilitar a comunicação entre a equipe de saúde; documentar as ações realizadas; testemunharas ações de enfermagem em processos legais e fornecer subsídios para a conduta diagnóstica e terapêutica ${ }^{(8)}$.

As dificuldades elencadas pelos enfermeiros desse estudo para a realização da consulta de enfermagem revelam a forma como organizam seu processo de trabalho em relação à implantação da consulta de enfermagem. A alegação da dificuldade de acesso aos protocolos/manuais pode ser refutada pelo fácil acesso desses materiais por meio da internet. Entretanto, a ausência de treinamento aponta para o não interesse da gestão na implantação da consulta de enfermagem e, mais especificamente, na SAE.

Enfermeiros de outro estudo também relataram dificuldades na implantação da SAE e na execução da técnica do exame físico, tais como: falta de conhecimento profissional; dificuldade no histórico de enfermagem com informações precisas; ausência de conhecimento na realização do exame físico; falta de tempo para planejar e colocar em prática as ações; falta de recursos materiais e excesso de atribuições do enfermeiro $^{(16)}$.

Poucos enfermeiros desse estudo conseguem realizar o diagnóstico de enfermagem. A maioria justifica a não existência da NANDA/CIPE. No âmbito da atenção básica, a Associação Brasileira de Enfermagem (ABEn) desenvolveu um projeto Classificação das Práticas de Enfermagem em Saúde Coletiva (CIPESC), entre 1996 a 2000, atendendo a uma proposta do Conselho Internacional de Enfermeiras (CIE), para a elaboração de um sistema que descrevesse a prática de enfermagem através de uma nomenclatura compartilhada pelos enfermeiros de todo o mundo ${ }^{(17)}$. A escassa publicação na área denota a incipiência e pouca divulgação da CIPESC no Brasil.

$\mathrm{O}$ estudo realizado em um hospital de Recife/PE sobre o conhecimento da SAE pelos enfermeiros constatou que $68 \%$ não citou nenhum diagnóstico e menos da metade conseguiram descrever todas as etapas da Sistematização, confirmando assim a falta de conhecimento ${ }^{(18)}$.

Ainda sobre essa etapa, em estudo desenvolvido sobre a análise da implementação da SAE constatou-se que os enfermeiros identificam mais diagnósticos ligados à área fisiológica do que os da área psicossocial. Esse fato está relacionado à resistência do enfermeiro em documentar diagnósticos nessas áreas, e também dificuldades de 
entendimento da terminologia da $\operatorname{NANDA}^{(12)}$.

$\mathrm{Na}$ etapa de planejamento, observou-se o uso de estratégias para garantia da adesão medicamentosa, mudança no estilo de vida e o autocuidado. Em relação à adesão medicamentosa, verificou-se em um estudo sobre a consulta de enfermagem ao paciente com hipertensão na Estratégia Saúde da Família, que o período mais curto entre uma consulta e outra estreita a relação entre os profissionais e pacientes, favorecendo, assim, a adesão ao tratamento ${ }^{(19)}$.

$\mathrm{O}$ uso do medicamento diariamente é eficaz e as modificações nos hábitos de vida resultando em alterações na forma de viver e na própria ideia de saúde e de qualidade de vida que o indivíduo possui, logo, a sensibilização e a educação do usuário se fazem necessárias para um adequado controle da doença ${ }^{(20)}$.

Em relação à implementação da SAE, os enfermeiros revelaram uma prática de enfermagem mais focada no modelo medicamentoso, visto que todos solicitam exames, mas poucos realizam atividades educativas. Ressalta-se que o grupo HIPERDIA tem como meta a orientação para o autocuidado, uma prática de atividades que as pessoas desempenham em seu próprio benefício $^{(21)}$. Para efetivar intervenções educativas junto a estes pacientes um caminho é formar grupos de portadores de DM e/ou HAS, a exemplo do grupo HIPERDIA, que possibilita a obtenção de informações acerca dos usuários e proporciona ao mesmo a obtenção de informações necessárias para o processo de educação em saúde e o alcance da melhoria da qualidade de vida ${ }^{(21)}$.

No que diz respeito à etapa de avaliação, a maioria dos enfermeiros avaliam $\mathrm{o}$ resultado de suas intervenções. Uma das funções do enfermeiro é a de solicitar os exames determinados pelo protocolo do MS. Quando não existirem intercorrências, repete-se a medicação, realiza-se a avaliação do "pé diabético", o controle da glicemia capilar, controle da HAS a cada consulta, além de avaliar os exames solicitados $^{(21)}$.

Em estudo sobre os aspectos contemplados na consulta de enfermagem ao usuário com hipertensão atendido no Programa Saúde da Família, todos os enfermeiros solicitavam exames complementares, mas nem todos verificavam os resultados dos exames anteriores ${ }^{(15)}$. Em caso de melhora deve-se avaliar o que foi feito, a fim de que possa apreender cada vez mais as melhores estratégias a serem adotadas. Em caso 
de piora ou manutenção do quadro, deve-se perguntar onde ocorreu falha, uma vez que a coleta de dados pode ter sido incompleta, o aprazamento para o alcance do resultado esperado pode ter sido equivocado ou as ações de enfermagem podem ter sido inadequadas $^{(11)}$.

\section{Conclusão}

Apesar de todos os profissionais que participaram da pesquisa afirmarem que realizam a consulta de enfermagem, percebe-se diversas limitações que impedem a utilização eficaz deste importante instrumento. Observa-se que os enfermeiros do estudo têm um bom ponto de partida, visto que todos realizam o exame físico; porém, na etapa seguinte, mais da metade dos enfermeiros investigados não consegue classificar o diagnóstico. Isso implica inferir que a SAE fica comprometida, pois é da etapa do diagnóstico que desencadeiam as demais.

Metade da amostra da atual pesquisa possui mais de dez anos de profissão, por isso justifica-se, em parte, a dificuldade da utilização da SAE na sua implementação do cuidado e, consequentemente, em estabelecer diagnósticos de enfermagem. Porém, a resolução do COREN que estabelece a obrigatoriedade da implatação da SAE nos serviços de saúde, data de 1993, tempo suficiente para a sua implantação nos serviços e ainda para a capacitação autodirigida por parte dos enfermeiros que devem se responsabilizar por sua competência técnica, segundo o que preconiza o seu códico de ética.

A pesquisa apresenta limitação pelo quantitativo da amostra, com apenas 10 enfermeiros, pois nos Centros de Saúde os enfermeiros são divididos por especialidades, com isso um número relativamente pequeno faz atendimento a esse usuários. Percebese ainda que a maioria trabalha com demanda espontânea e não consegue fixar o grupo de HIPERDIA, pelo fato de não existir uma Equipe de Saúde da Família que realize um acompanhamento domiciliar, sendo um atendimento exclusivo no Centro através da demanda do usuário.

\section{Referências}

1. Sociedade Brasileira de Cardiologia, Sociedade Brasileira de Hipertensão, Sociedade Brasileira de Nefrologia. VI Diretrizes brasileiras de hipertensão. (São Paulo) [periódico na internet]. 2010 [citado 2015 mar. 25]; 95(1):1-51. Disponível em: http:// www.scielo.br/pdf/abc/v95n1s1/v95n1 s1.pdf. 
2. Freitas LR, Garcia LP. Evolução da prevalência do diabetes e deste associado à hipertensão arterial no Brasil: Análise da Pesquisa Nacional por Amostra de Domicílios, 1998, 2003 e 2008. Epidemiol. Serv. Saúde (Brasília) [periódico na internet]. 2012 [citado 2015 mar. 30]; 21(1): 7-19. Disponível em: http://scielo.iec.pa.gov .br/pdf/ess/v21n1/v21n1a02.pdf

3. Ministério da Saúde (BR). Departamento de Atenção Básica. Área Técnica de

Diabetes e Hipertensão Arterial. Hipertensão arterial sistêmica (HAS) e Diabetes mellitus (DM): protocolo. Caderno de Atenção Básica 7 Brasília: Ministério da Saúde; 2001.

4. Amaral LR, Oliveira MAD, Cardoso RB, Ávila SPAR. Atuação do enfermeiro como educador no programa saúde da família: importância para uma abordagem integral na atenção primária. Enfermagem (Guanambi) [periódico na internet]. 2011 [citado 2015 abr. 3]; 1(1): 1-21. Disponível em: http://www3.pgenf.ufba.br/SEMINARI O/ANAIS $/ 3 \% 20$ Educacao $\% 20 \mathrm{em} \% 20$ enfermagem/ATUACAO $\% 20 \mathrm{DO} \% 20$ ENFERMEIRO\%20COMO.pdf.

5. Borges JWP, Pinheiro NMG, Souza AC. Hipertensão comunicada e hipertensão compreendida: saberes e práticas de enfermagem em um Programa de Saúde da família de Fortaleza. (Ceará) [periódico na internet]. 2012 [citado 2015 abr. 15]; 7(1): 179-89. Disponível em: http://www.scielo.br/pdf/csc/v17n1/a2 0v17n1.pdf.
6. Conselho Federal de Enfermagem (BR). Resolução $\mathrm{N}^{\circ} 159$, de 19 de abril de 1993. Dispõe sobre a consulta de enfermagem. Rio de Janeiro: COFEN; 1993. Disponível em:http://www.cofen .gov.br/resoluo-cofen-1591993_4241. html.

7. Nascimento VF. Três instrumentos utilizados na sistematização da assistência de enfermagem em adultos na atenção básica. (Brasília) [periódico na internet]. 2013 [citado 2015 abr. 30]; 4(3): 1220-34. Disponível em: http://periodicos.unb.br/index.php/rgs/i ndex/index.php/gestaoesaude/article/vi ew/512.

8. Oliveira SKP, Queiróz APO, Matos DPMM, Moura AF, Lima FET. Temas abordados na consulta de enfermagem: Revisão integrativa da literatura. Enfermagem (Brasília) [periódico na internet]. 2012 [citado 2015 mai. 2]; 65(1): 155-61. Disponível em: http://www.scielo.br/scielo.php?script $=$ sci_arttext\&pid=S0034-71672012000 100023 .

9. Potter PA, PerryAG.Necessidades humanas básicas. In: Nascimento MIC (tradutora). Fundamentos de Enfermagem. Rio de Janeiro: Elsevier; $2013 ; 8$.

10. Diagnósticos de Enfermagem da NANDA: Definições e classificação 2009-2011. NANDA internacional. Tradução: Regina Machado Garcez. Porto Alegre: Artmed; 2010.

11. Tannure MC, Gonçalves AMP. SAE - Sistematização da Assistência de Enfermagem: guia prático. Rio de Janeiro: Guanabara Koogan; 2010;2. 
12. Neves RS, Shimizu HE. Análise da implementação da Sistematização da Assistência de Enfermagem em uma unidade de Reabilitação. Enfermagem (Brasília) [periódico na internet]. 2010 [citado 2015 mai. 6]; 63(2): 222-9. Disponível em: http://www.scielo.br/ pdf/reben/v63n2/09.

13. Nóbrega ESL, Medeiros ALF, Leite MCA. Atuação do Enfermeiro no controle da Hipertensão Arterial em Unidades de Saúde da Família. Enfermagem (Pernambuco) [periódico na internet]. 2010 [citado 2015 mai. 14]; 4(1): 50-60. Disponível em: https://periodicos.ufpe.br/revistas/revis taenfermagem/article/viewFile/5832/5 089.

14. Machado MH, Vieira ALS, Oliveira E. Construindo o perfil da enfermagem. Enfermagem em Foco. Enfermagem (Bahia) [periódico na internet]. 2012 [citado 2015 mai. 14]; 3(3): 119-22. Disponível em: http://revista.portalcofen.gov.br/index. php/enfermagem/article/view/294/156.

15. Moura DJM, Freitas MC, Guedes MVC, Lopes MVO, Menezes LCG, Barros AA. Sistematização da assistência de enfermagem fundamentada na CIPE e na teoria da adaptação em hipertensos. Enfermagem (Goiás) [periódico na internet]. 2014 [citado 2015 mai. 20]; 16(4): 710-9. Disponível em: https://www.fen.ufg.br/fen_revista/v16 /n4/pdf/v16n4a02.pdf.

16. Remizoski J, Rocha MM, Vall J. Dificuldades na implementação da assistência de enfermagem - SAE: uma revisão teórica. Enfermagem (Curitiba) [periódico na internet]. 2010 [citado 2015 mai. 25]; 3: 1-14. Disponível em: http://revistas.unibrasil .com.br/cadernossaude/index.php/saud e/article/viewFile/68/68.
17. Egry EY, Antunes MJM, Lopes MGD. Projeto CIPESC CIE-ABEn. IN: Garcia TR, Egry EY. Integralidade da atenção no SUS e a Sistematização da Assistência de Enfermagem. Porto Alegre: Artmed; 2010:175-91.

18. Silva EGC, Oliveira VC, Neves GBC, Guimarães TMR. O conhecimento do enfermeiro sobre a Sistematização da Assistência de Enfermagem: da teoria à prática. Enfermagem (São Paulo) [periódico na internet]. 2011 [citado 2015 mai. 31]; 45(6): 1380-6. Disponível em: http://www.scielo.br/scielo.php?script $=$ sci_arttext\&pid $=$ S0080-6234201100 0600015 .

19. Branco CSN, Mendes RS, Oliveira SKP, Pamplona YAP. Consulta de enfermagem ao paciente com hipertensão na estratégia de saúde da família. Enfermagem (Bahia) [periódico na internet]. 2013 [citado 2015 jun. 8]; 2(1): 196-8. Disponível em: https://www5.bahiana.edu.br/index.ph p/enfermagem/article/view/232/228.

20. Andrade LDF, Carvalho PMD, Pinto MB, Santos NLCB, Lima EAR. Considerações sobre a consulta de enfermagem: o olhar de hipertensos da atenção pública primária no município de Petrolina/ PE. Enfermagem (Rio Verde) [periódico na internet]. 2013 [citado 2015 jun. 14]; 11(2): 11-1. Disponível em: http://periodicos. unincor.br/index.php/revistaunincor/art icle/view/1078.

21. Carvalho CG. Assistência de enfermagem aos portadores de hipertensão arterial sistêmica e Diabetes Mellitus: educação em saúde no grupo Hiperdia. Enfermagem (Rio Verde) [periódico na internet]. 2012 [citado 2015 jun. 26]; 5(1): 39-6. Disponível em: http://revistas.unibh.br/ index.php/dcbas/article/view/201/466. 